\title{
Retrieving the Elastodynamic Green's Function of an Arbitrary Inhomogeneous Medium by Cross Correlation
}

\author{
Kees Wapenaar* \\ Department of Geotechnology, Delft University of Technology, P.O. Box 5028, 2600 GA Delft, The Netherlands
}

(Received 18 August 2004; published 16 December 2004)

\begin{abstract}
A correlation-type reciprocity theorem is used to show that the elastodynamic Green's function of any inhomogeneous medium (random or deterministic) can be retrieved from the cross correlation of two recordings of a wave field at different receiver locations at the free surface. Unlike in other derivations, which apply to diffuse wave fields in random media or irregular finite bodies, no assumptions are made about the diffusivity of the wave field. In a second version, it is assumed that the wave field is diffuse due to many uncorrelated sources inside the medium.
\end{abstract}

DOI: 10.1103/PhysRevLett.93.254301

PACS numbers: $43.20 .+\mathrm{g}, 43.60 .+\mathrm{d}, 91.30 .-\mathrm{f}$

Recently it has been shown by various authors that the Green's function of a random medium or an irregular finite body can be obtained by cross correlating the recordings of a diffuse wave field at two receiver positions [1-5]. The resulting Green's function is the wave field that would be observed at one of these receiver positions if there were an impulsive source at the other. This theoretical result has been successfully demonstrated with ultrasonic measurements [4-7] and seismic surface waves $[8,9]$. The accuracy of the reconstructed Green's function depends on the amount of disorder of the medium parameters and the duration of the signal. Ideally the cross correlations should be done in the equipartitioned regime (where the net energy flux is equal to zero), which takes place after sufficiently long multiple scattering of the wave field between the heterogeneities in the disordered medium [7].

An initially independent line of research deals with the reconstruction of the seismic reflection response of a deterministic medium from passive recordings of the transmission response. Already in 1968 Claerbout showed that the autocorrelation of the transmission response of a horizontally layered earth yields the superposition of the reflection response and its time-reversed version [10]. The source in the subsurface may be a transient or a noise signal; in both cases the source signature in the reconstructed reflection response is the autocorrelation of the source signal in the subsurface. This method has been applied to microearthquake data [11]. The derivation in [10] was strictly one dimensional. Later Claerbout conjectured for the 3D situation that "by cross-correlating noise traces recorded at two locations on the surface, we can construct the wave field that would be recorded at one of the locations if there was a source at the other" [12]. Although it was not explicitly stated, this conjecture applies to deterministic media: in exploration seismology the earth is usually considered to be built up of geological layers with smoothly varying properties, separated by well-defined curved interfaces and faults which act as the main reflectors; scattering due to disorder of the parameters within the geological layers is generally considered a second order effect. Numerical modeling studies have been carried out to confirm Claerbout's conjecture [13]. These modeling studies showed that "longer time series, and a white spatial distribution of random noise events would be necessary for the conjecture to work in practice." The crosscorrelation approach has been applied successfully to helioseismic data $[12,14]$. Recently Claerbout's conjecture has been proven by the author $[15,16]$. The proof also explains the empirical observations of the numerical modeling studies.

In this Letter we derive a relation between the elastodynamic Green's function and the cross correlation of observed wave fields that holds at the free surface of random as well as deterministic media. The approach is quite different from that in [1-7], which holds only for diffuse wave fields. It is also different from the derivation in $[15,16]$, which is based on coupled one-way wave equations for acoustic down-going and up-going waves, assumes a certain degree of smoothness of the medium parameters, and ignores evanescent waves.

The basis for our derivation is a reciprocity theorem, which relates two independent elastodynamic states (wave fields and sources) in one and the same medium [17]. One can distinguish between reciprocity theorems of the convolution type and of the correlation type [18]. Correlation-type reciprocity theorems contain correlations between the wave fields and sources in both states. Since it is our aim to retrieve the Green's function from the cross correlation of observed wave fields, the correlation-type reciprocity theorem is a natural choice.

Let an elastodynamic wave field be characterized by the space- and time-dependent particle velocity $v_{i}(\mathbf{x}, t)$ and stress tensor $\tau_{i j}(\mathbf{x}, t)$. Here $\mathbf{x}=\left(x_{1}, x_{2}, x_{3}\right)$ and $t$ denote the Cartesian coordinate vector and time, respectively. Subscripts $i$ and $j$ take on the values 1,2, and 3. We use Einstein's summation convention for repeated lower- 
case subscripts. We define the temporal Fourier transform of a space- and time-dependent quantity as $\hat{v}_{i}(\mathbf{x}, \omega)=$ $\int \exp (-j \omega t) v_{i}(\mathbf{x}, t) d t$, where $j$ is the imaginary unit and $\omega$ the angular frequency. In the space-frequency domain the particle velocity and stress tensor in an inhomogeneous anisotropic lossless medium obey the equation of motion $j \omega \rho \hat{v}_{i}-\partial_{j} \hat{\tau}_{i j}=\hat{f}_{i}$ and the stress-strain relation $-j \omega s_{i j k l} \hat{\tau}_{k l}+\left(\partial_{j} \hat{v}_{i}+\partial_{i} \hat{v}_{j}\right) / 2=\hat{h}_{i j}$, where $\partial_{j}$ is the partial derivative in the $x_{j}$ direction, $\rho(\mathbf{x})$ the mass density of the medium, $s_{i j k l}(\mathbf{x})$ its compliance, $\hat{f}_{i}(\mathbf{x}, \omega)$ the external volume force, and $\hat{h}_{i j}(\mathbf{x}, \omega)$ the external deformation rate. We consider the "interaction quantity" $\hat{v}_{i, A}^{*} \hat{\tau}_{i j, B}+$ $\hat{\tau}_{i j, A}^{*} \hat{v}_{i, B}$, where the asterisk $*$ denotes complex conjugation and subscripts $A$ and $B$ are used to distinguish the two independent states. Note that the product $\hat{v}_{i, A}^{*} \hat{\tau}_{i j, B}$ is the Fourier transform of the cross correlation of $v_{i, A}(\mathbf{x}, t)$ and $\tau_{i j, B}(\mathbf{x}, t)$; a similar remark holds for $\hat{\tau}_{i j, A}^{*} \hat{v}_{i, B}$. The elastodynamic reciprocity theorem of the correlation type is obtained by applying the operator $\partial_{j}$ to the interaction quantity, substituting the equation of motion and the stress-strain relation for states $A$ and $B$, using the symmetry relations $\hat{\tau}_{i j}=\hat{\tau}_{j i}$ and $s_{i j k l}=s_{k l i j}$, integrating the result over a spatial domain $\mathbb{D}$ with boundary $\partial \mathbb{D}$ and outward pointing normal vector $\mathbf{n}=\left(n_{1}, n_{2}, n_{3}\right)$, and applying the theorem of Gauss [17]. This gives

$$
\begin{aligned}
\oint_{\partial \mathbb{D}}\left\{\hat{\boldsymbol{v}}_{i, A}^{*} \hat{t}_{i, B}+\hat{t}_{i, A}^{*} \hat{\boldsymbol{v}}_{i, B}\right\} d^{2} \mathbf{x}= & \int_{\mathbb{D}}\left\{\hat{h}_{i j, A}^{*} \hat{\tau}_{i j, B}-\hat{f}_{i, A}^{*} \hat{\boldsymbol{v}}_{i, B}\right. \\
& \left.+\hat{\tau}_{i j, A}^{*} \hat{h}_{i j, B}-\hat{v}_{i, A}^{*} \hat{f}_{i, B}\right\} d^{3} \mathbf{x},
\end{aligned}
$$

with the traction $\hat{t}_{i}$ at the boundary $\partial \mathbb{D}$ defined as $\hat{t}_{i}=$ $\hat{\tau}_{i j} n_{j}$. We apply this theorem to the wave fields in an arbitrary inhomogeneous anisotropic lossless medium, bounded by a free surface (Fig. 1). We choose $\partial \mathbb{D}$ such that it consists of a part of the free surface, denoted by $\partial \mathbb{D}_{0}$, and an arbitrarily shaped surface $\partial \mathbb{D}_{1}$ inside the medium. Furthermore, we assume that the sources $\hat{h}_{i j, A}$, $\hat{f}_{i, A}, \hat{h}_{i j, B}$, and $\hat{f}_{i, B}$ in $\mathbb{D}$ are zero. Hence, Eq. (1) becomes

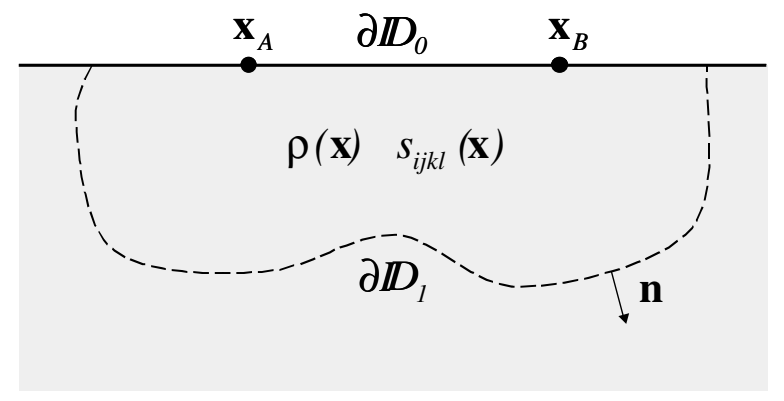

FIG. 1. Inhomogeneous anisotropic lossless medium, bounded by a free surface.

$$
\begin{aligned}
\int_{\partial \mathbb{D}_{0}}\left\{\hat{\boldsymbol{v}}_{i, A}^{*} \hat{t}_{i, B}+\hat{t}_{i, A}^{*} \hat{\boldsymbol{v}}_{i, B}\right\} d^{2} \mathbf{x}= & -\int_{\partial \mathbb{D}_{1}}\left\{\hat{\boldsymbol{v}}_{i, A}^{*} \hat{t}_{i, B}\right. \\
& \left.+\hat{t}_{i, A}^{*} \hat{\boldsymbol{v}}_{i, B}\right\} d^{2} \mathbf{x} .
\end{aligned}
$$

We introduce sources in terms of boundary conditions at the free surface $\partial \mathbb{D}_{0}$. This is possible since at a free surface the traction is zero everywhere, except at those positions where a source traction is applied. In state $A$ we apply a source traction in the $x_{p}$ direction at $\mathbf{x}_{A} \in \partial \mathbb{D}_{0}$ with source function $s(t)$. Hence, in the space-frequency domain the traction for $\mathbf{x} \in \partial \mathbb{D}_{0}$ is given by $\hat{t}_{i, A}(\mathbf{x}, \omega)=$ $\delta\left(\mathbf{x}-\mathbf{x}_{A}\right) \delta_{i p} \hat{s}(\omega)$, where $\delta\left(\mathbf{x}-\mathbf{x}_{A}\right)$ is a 2D Dirac delta function and $\delta_{i p}$ is the Kronecker delta function. The observed particle velocity at $\mathbf{x}$ due to the source at $\mathbf{x}_{A}$ is expressed as $\hat{\boldsymbol{v}}_{i, A}(\mathbf{x}, \omega)=\hat{G}_{i, p}^{v, t}\left(\mathbf{x}, \mathbf{x}_{A}, \omega\right) \hat{s}(\omega)$, where $\mathbf{x}$ can be anywhere at the free surface or in the medium. $\hat{G}_{i, p}^{v, t}\left(\mathbf{x}, \mathbf{x}_{A}, \omega\right)$ represents a Green's function, with the following notation convention: the two coordinate vectors between the brackets (here $\mathbf{x}$ and $\mathbf{x}_{A}$ ) represent the observation point and the source point, respectively; the superscripts (here $v$ and $t$ ) represent the observed quantity (velocity) and the source quantity (traction), respectively; the subscripts (here $i$ and $p$ ) represent the component of the observed quantity and the source quantity, respectively. Similarly, in state $B$ we apply a source traction in the $x_{q}$ direction at $\mathbf{x}_{B} \in \partial \mathbb{D}_{0}$ with the same source function $s(t)$. Hence, $\hat{t}_{i, B}(\mathbf{x}, \omega)=\delta\left(\mathbf{x}-\mathbf{x}_{B}\right) \delta_{i q} \hat{s}(\omega)$ for $\mathbf{x} \in$ $\partial \mathbb{D}_{0}$ and $\hat{\boldsymbol{v}}_{i, B}(\mathbf{x}, \omega)=\hat{G}_{i, q}^{v, t}\left(\mathbf{x}, \mathbf{x}_{B}, \omega\right) \hat{s}(\omega)$ for any $\mathbf{x}$. Substituting these expressions in the left-hand side of Eq. (2), and using reciprocity of the Green's function [i.e., $\hat{G}_{q, p}^{v, t}\left(\mathbf{x}_{B}, \mathbf{x}_{A}, \omega\right)=\hat{G}_{p, q}^{v, t}\left(\mathbf{x}_{A}, \mathbf{x}_{B}, \omega\right)$ ], yields

$$
\begin{aligned}
\int_{\partial \mathbb{D}_{0}}\left\{\hat{v}_{i, A}^{*} \hat{t}_{i, B}+\hat{t}_{i, A}^{*} \hat{v}_{i, B}\right\} d^{2} \mathbf{x}= & {\left[\left\{\hat{G}_{q, p}^{v, t}\left(\mathbf{x}_{B}, \mathbf{x}_{A}, \omega\right)\right\}^{*}\right.} \\
& \left.+\hat{G}_{p, q}^{v, t}\left(\mathbf{x}_{A}, \mathbf{x}_{B}, \omega\right)\right]|\hat{s}(\omega)|^{2} \\
= & 2 \operatorname{Re}\left\{\hat{G}_{p, q}^{v, t}\left(\mathbf{x}_{A}, \mathbf{x}_{B}, \omega\right)\right\}|\hat{s}(\omega)|^{2}
\end{aligned}
$$

where $\operatorname{Re}\{\}$ denotes the real part. In order to evaluate the right-hand side of Eq. (2), we note that in states $A$ and $B$ the velocities and tractions for $\mathbf{x} \in \partial \mathbb{D}_{1}$, due to the traction sources at $\mathbf{x}_{A}$ and $\mathbf{x}_{B}$, can be expressed as

$$
\begin{aligned}
\hat{v}_{i, A}(\mathbf{x}, \omega) & =\hat{G}_{i, p}^{v, t}\left(\mathbf{x}, \mathbf{x}_{A}, \omega\right) \hat{s}(\omega)=\hat{G}_{p, i}^{v, f}\left(\mathbf{x}_{A}, \mathbf{x}, \omega\right) \hat{s}(\omega), \\
\hat{t}_{i, A}(\mathbf{x}, \omega) & =\hat{G}_{i, p}^{t, t}\left(\mathbf{x}, \mathbf{x}_{A}, \omega\right) \hat{s}(\omega)=\hat{G}_{p, i}^{v, h}\left(\mathbf{x}_{A}, \mathbf{x}, \omega\right) \hat{s}(\omega), \\
\hat{v}_{i, B}(\mathbf{x}, \omega) & =\hat{G}_{i, q}^{v, t}\left(\mathbf{x}, \mathbf{x}_{B}, \omega\right) \hat{s}(\omega)=\hat{G}_{q, i}^{v, f}\left(\mathbf{x}_{B}, \mathbf{x}, \omega\right) \hat{s}(\omega), \\
\hat{t}_{i, B}(\mathbf{x}, \omega) & =\hat{G}_{i, q}^{t, t}\left(\mathbf{x}, \mathbf{x}_{B}, \omega\right) \hat{s}(\omega)=\hat{G}_{q, i}^{v, h}\left(\mathbf{x}_{B}, \mathbf{x}, \omega\right) \hat{s}(\omega) .
\end{aligned}
$$

Note that the second Green's function in each of these equations is the reciprocal of the first and can therefore be interpreted as an observation at the free surface (at $\mathbf{x}_{A}$ or $\left.\mathbf{x}_{B}\right)$, due to a source at $\mathbf{x} \in \partial \mathbb{D}_{1}$. In $\hat{G}_{p, i}^{v, f}\left(\mathbf{x}_{A}, \mathbf{x}, \omega\right)$ the observed quantity is velocity and the source is a volume 
force; in $\hat{G}_{p, i}^{v, h}\left(\mathbf{x}_{A}, \mathbf{x}, \omega\right)$ the observed quantity is again velocity but the source is a specific type of deformation rate source (actually $\hat{G}_{p, i}^{v, h}=n_{j} \hat{G}_{p, i j}^{v, h}$, where the source for $\hat{G}_{p, i j}^{v, h}$ is a deformation rate tensor and $n_{j}$ is the $j$ component of the normal at $\partial \mathbb{D}_{1}$ ). Substituting these expressions in the right-hand side of Eq. (2) (and using Eq. (3) for the left-hand side) we obtain

$$
2 \operatorname{Re}\left\{\hat{G}_{p, q}^{v, t}\left(\mathbf{x}_{A}, \mathbf{x}_{B}, \omega\right)\right\}=-\int_{\partial \mathbb{D}_{1}}\left[\left\{\hat{G}_{p, i}^{v, f}\left(\mathbf{x}_{A}, \mathbf{x}, \omega\right)\right\}^{*} \hat{G}_{q, i}^{v, h}\left(\mathbf{x}_{B}, \mathbf{x}, \omega\right)+\left\{\hat{G}_{p, i}^{v, h}\left(\mathbf{x}_{A}, \mathbf{x}, \omega\right)\right\}^{*} \hat{G}_{q, i}^{v, f}\left(\mathbf{x}_{B}, \mathbf{x}, \omega\right)\right] d^{2} \mathbf{x}
$$

The left-hand side of this equation represents the Fourier transform of the superposition of the time domain Green's function $G_{p, q}^{v, t}\left(\mathbf{x}_{A}, \mathbf{x}_{B}, t\right)$ and its time-reversed version at the free surface. Since the Green's function is causal, it does not interfere with its time-reversed version (except at $t=0$ ), so it is recovered by multiplying the two-sided result with the Heaviside step function $H(t)$. Note that this Green's function represents the velocity in the $x_{p}$ direction at $\mathbf{x}_{A}$ due to a source traction in the $x_{q}$ direction at $\mathbf{x}_{B}$. The terms under the integral on the righthand side represent cross correlations of particle velocities in the $x_{p}$ and $x_{q}$ directions at $\mathbf{x}_{A}$ and $\mathbf{x}_{B}$, respectively, due to volume force and deformation rate sources at $\mathbf{x}$ on an arbitrarily shaped surface $\partial \mathbb{D}_{1}$ in the medium. Equation (4) is exact, so in theory it is possible to retrieve the exact Green's function, including the coda, of any inhomogeneous anisotropic lossless medium (random or deterministic) from cross correlations of observed particle velocities at the free surface. Unlike in the approach for diffuse wave fields [1-7], which requires only one or a few sources, in Eq. (4) it is assumed that volume force and deformation rate sources are available everywhere on $\partial \mathbb{D}_{1}$ and that the response of each source is measured separately. In general not all sources are equally important. When the aim is to retrieve surface waves, the main contribution comes from sources at $\partial \mathbb{D}_{1}$ close to the free surface. On the other hand, in order to retrieve the reflection response, sources at $\partial \mathbb{D}_{1}$ below $\mathbf{x}_{A}$ and $\mathbf{x}_{B}$ give the main contribution.

In the following we make some approximations which circumvent several of the assumptions mentioned above. When the medium outside $\partial \mathbb{D}_{1}$ is homogeneous and source-free, Eq. (4) may be approximated by

$$
2 \operatorname{Re}\left\{\hat{G}_{p, q}^{v, t}\left(\mathbf{x}_{A}, \mathbf{x}_{B}, \omega\right)\right\} \approx-\int_{\partial \mathbb{D}_{1}}\left\{\hat{G}_{p, k}^{v, \phi}\left(\mathbf{x}_{A}, \mathbf{x}, \omega\right)\right\}^{*} \hat{G}_{q, k}^{v, \phi}\left(\mathbf{x}_{B}, \mathbf{x}, \omega\right) d^{2} \mathbf{x}
$$

which follows from an analysis similar as in [19]. The Green's functions in the right-hand side represent again velocities at $\mathbf{x}_{A}$ and $\mathbf{x}_{B}$ due to sources at $\mathbf{x} \in \partial \mathbb{D}_{1}$. The superscript $\phi$ denotes that these sources are quasi $P$-wave sources (for $k=1$ ) and quasi $S$-wave sources with different polarizations (for $k=2,3$ ). The accuracy of Eq. (5) depends on the curvature of $\partial \mathbb{D}_{1}$ (when $\partial \mathbb{D}_{1}$ would be planar, the only approximation would be that evanescent waves are neglected). Next we assume mutually uncorrelated noise sources $\hat{N}_{k}(\mathbf{x}, \omega)$ and $\hat{N}_{l}\left(\mathbf{x}^{\prime}, \omega\right)$ for any $\mathbf{x}$ and $\mathbf{x}^{\prime}$ at $\partial \mathbb{D}_{1}$, obeying the relation $\left\langle\hat{N}_{k}^{*}(\mathbf{x}, \omega) \hat{N}_{l}\left(\mathbf{x}^{\prime}, \omega\right)\right\rangle=$ $\delta_{k l} \delta\left(\mathbf{x}-\mathbf{x}^{\prime}\right) \hat{S}(\omega)$, where \langle\rangle denotes a spatial ensemble average and $\hat{S}(\omega)$ the power spectrum of the noise (which is assumed to be the same for all sources). Inserting this relation in the right-hand side of Eq. (5) yields

$$
2 \operatorname{Re}\left\{\hat{G}_{p, q}^{v, t}\left(\mathbf{x}_{A}, \mathbf{x}_{B}, \omega\right)\right\} \hat{S}(\omega) \approx-\left\langle\left\{\hat{\boldsymbol{v}}_{p}^{\mathrm{obs}}\left(\mathbf{x}_{A}, \omega\right)\right\}^{*} \hat{\boldsymbol{v}}_{q}^{\mathrm{obs}}\left(\mathbf{x}_{B}, \omega\right)\right\rangle,
$$

where

$$
\hat{\boldsymbol{v}}_{p}^{\mathrm{obs}}\left(\mathbf{x}_{A}, \omega\right)=\int_{\partial \mathbb{D}_{1}} \hat{G}_{p, k}^{v, \phi}\left(\mathbf{x}_{A}, \mathbf{x}, \omega\right) \hat{N}_{k}(\mathbf{x}, \omega) d^{2} \mathbf{x}
$$

and

$$
\hat{v}_{q}^{\mathrm{obs}}\left(\mathbf{x}_{B}, \omega\right)=\int_{\partial \mathbb{D}_{1}} \hat{G}_{q, l}^{v, \phi}\left(\mathbf{x}_{B}, \mathbf{x}^{\prime}, \omega\right) \hat{N}_{l}\left(\mathbf{x}^{\prime}, \omega\right) d^{2} \mathbf{x}^{\prime}
$$

Here $\hat{v}_{p}^{\text {obs }}\left(\mathbf{x}_{A}, \omega\right)$ and $\hat{v}_{q}^{\text {obs }}\left(\mathbf{x}_{B}, \omega\right)$ are the observed particle velocities at $\mathbf{x}_{A}$ and $\mathbf{x}_{B}$ at the free surface due to a distribution of noise sources at an arbitrarily shaped surface $\partial \mathbb{D}_{1}$ inside the medium. The average in Eq. (6) is taken over different realizations of the source distribution. In the time domain Eq. (6) becomes

$$
\begin{aligned}
\int_{-\infty}^{\infty}\left\{G_{p, q}^{v, t}\left(\mathbf{x}_{A}, \mathbf{x}_{B},-t^{\prime}\right)+\right. & \\
\left.G_{p, q}^{v, t}\left(\mathbf{x}_{A}, \mathbf{x}_{B}, t^{\prime}\right)\right\} S\left(t-t^{\prime}\right) d t^{\prime} \approx & -\left\langle\int_{-\infty}^{\infty} v_{p}^{\mathrm{obs}}\left(\mathbf{x}_{A}, t+t^{\prime}\right)\right. \\
& \left.\times v_{q}^{\mathrm{obs}}\left(\mathbf{x}_{B}, t^{\prime}\right) d t^{\prime}\right\rangle
\end{aligned}
$$

According to this equation, the cross correlation of the observed particle velocities at $\mathbf{x}_{A}$ and $\mathbf{x}_{B}$ yields the elastodynamic Green's function between $\mathbf{x}_{A}$ and $\mathbf{x}_{B}$, convolved with the autocorrelation of the noise sources. The advantage of Eq. (9) over Eq. (4) is that no separate measurements of the responses of all sources at $\partial \mathbb{D}_{1}$ are required; these responses can be measured simultaneously, according to Eqs. (7) and (8). Note that by omit- 
ting the subscripts $p, q, k$, and $l$ in Eqs. (7)-(9) we obtain an expression for the acoustic Green's function at the free surface of an inhomogeneous lossless fluid.

Equation (9) has a striking resemblance to the result of [1-7]. In Eq. (9) there are no specific assumptions made about the medium, but there should be many mutually uncorrelated sources present, which leads again to a diffuse wave field.

An application of Eq. (9) is passive seismic imaging. In this application $v_{p}^{\text {obs }}\left(\mathbf{x}_{A}, t\right)$ and $v_{q}^{\text {obs }}\left(\mathbf{x}_{B}, t\right)$ represent passive recordings by geophones at the earth's surface of noise generated by natural sources in the subsurface. By placing many geophones at the surface, the reflection response can be reconstructed for many $\mathbf{x}_{A}$ 's and $\mathbf{x}_{B}$ 's. By downward extrapolating these responses into the subsurface one can subsequently form an image of the subsurface [20-24]. This procedure makes no assumptions with respect to the diffusivity of the wave field. Suppose that the medium is fully deterministic and that there is only one noise source present in the subsurface. Although for this situation it is not possible to reconstruct the Green's function as an intermediate result, the passive imaging method still maps the primary reflection response (i.e., the ballistic wave) to its correct scattering origin in depth as long as the specular reflection point at the free surface lies within the array of geophones. This has been shown with stationary phase analysis [20-22] and confirmed with numerical modeling studies [24].

In conclusion, we have shown that the elastodynamic Green's function of any inhomogeneous medium (random or deterministic) can be retrieved from the cross correlation of two recordings of a wave field at different receiver locations. Unlike in the derivations in [1-7], which apply to diffuse wave fields in random media or finite bodies, we have made no assumptions about the distribution of the medium parameters. Using a reciprocity theorem of the correlation type, we derived an exact representation for the Green's function in terms of an integral of cross correlations of observed wave fields at two points at the free surface [Eq. (4)]; the integral is along an arbitrarily shaped surface inside the medium, which contains the sources of the observed wave fields. Next we assumed that these sources are mutually uncorrelated noise sources, which led to an expression [Eq. (9)] analogous to that in [1-7]. Finally, we indicated that for passive imaging of the ballistic wave the wave field need not be diffusive at all: a single source in a deterministic medium suffices, as long as the receiver array covers the specular reflection point at the free surface [20-22].

This work is supported by the Netherlands Research Centre for Integrated Solid Earth Science (ISES). I thank my colleagues Deyan Draganov, Evert Slob, and Jesper
Spetzler for carefully reading the manuscript.

*Electronic address: C.P.A.Wapenaar@CiTG.TUDelft.NL

[1] R. L. Weaver and O. I. Lobkis, Phys. Rev. Lett. 87, 134301 (2001).

[2] B. A. van Tiggelen, Phys. Rev. Lett. 91, 243904 (2003).

[3] R. K. Snieder, Phys. Rev. E 69, 046610 (2004).

[4] O. I. Lobkis and R. L. Weaver, J. Acoust. Soc. Am. 110, 3011 (2001).

[5] R. L. Weaver and O. I. Lobkis, Ultrasonics 40, 435 (2002).

[6] A. Derode, E. Larose, M. Tanter, J. de Rosny, A. Tourin, M. Campillo, and M. Fink, J. Acoust. Soc. Am. 113, 2973 (2003).

[7] A. E. Malcolm, J. A. Scales, and B. A. van Tiggelen, Phys. Rev. E 70, 015601 (2004).

[8] M. Campillo and A. Paul, Science 299, 547 (2003).

[9] N. M. Shapiro and M. Campillo, Geophys. Res. Lett. 31, L07614 (2004).

[10] J. F. Claerbout, Geophysics 33, 264 (1968).

[11] M. R. Daneshvar, C.S. Clay, and M. K. Savage, Geophysics 60, 1178 (1995).

[12] J. Rickett and J. Claerbout, Leading Edge 18, 957 (1999).

[13] J. Rickett and J. F. Claerbout, Stanford Exploration Project Technical Report, 1996.

[14] T. L. Duvall, S. M. Jefferies, J.W. Harvey, and M. A. Pomerantz, Nature (London) 362, 430 (1993).

[15] C. P. A. Wapenaar, D. Draganov, J.W. Thorbecke, and J. T. Fokkema, in Expanded Abstracts of the 72nd Annual Meeting of the Society of Exploration Geophysicists (Society of Exploration Geophysicists, Tulsa, OK, 2002).

[16] C. P. A. Wapenaar, Geophysics 68, 1756 (2003).

[17] A. T. de Hoop, Appl. Sci. Res. 16, 39 (1966).

[18] N. N. Bojarski, J. Acoust. Soc. Am. 74, 281 (1983).

[19] C. P. A. Wapenaar and G. C. Haimé, Geophys. Prospect. 38, 23 (1990).

[20] G. T. Schuster, in Extended Abstracts of the 63rd Annual Meeting of the European Association of Geoscientists and Engineers (European Association of Geoscientists and Engineers, Houten, The Netherlands, 2001).

[21] G. T. Schuster, J. Yu, J. Sheng, and J. Rickett, Geophys. J. Int. 157, 838 (2004).

[22] C. P. A. Wapenaar, D. Draganov, J. van der Neut, and J.W. Thorbecke, in Expanded Abstracts of the 74th Annual Meeting of the Society of Exploration Geophysicists (Society of Exploration Geophysicists, Tulsa, OK, 2004).

[23] B. Artman, D. Draganov, C. P. A. Wapenaar, and B. Biondi, in Extended Abstracts of the 66th Annual Meeting of the European Association of Geoscientists and Engineers (European Association of Geoscientists and Engineers, Houten, The Netherlands, 2004).

[24] D. Draganov, C. P. A. Wapenaar, B. Artman, and B. Biondi, in Expanded Abstracts of the 74th Annual Meeting of the Society of Exploration Geophysicists (Ref. [22]). 